\title{
Passive high ratio sunlight concentration configurations
}

\section{A. Rudnitsky}

A. Zaban

Z. Zalevsz@biu.ac.il
Faculty of Engineering, Bar-Ilan University, Ramat-Gan 52900, Israel

Dept. of Chemistry, Bar-Ilan University, Ramat-Gan 52900, Israel

Faculty of Engineering, Bar-Ilan University, Ramat-Gan 52900, Israel

During the day the sun is moving along curved 1-D trajectory across the sky. This is essential a priori information that can be used in order to design passive optics to perform efficient collection of sunlight without tracking after sun's location in the sky. Despite the movement of the sun along its trajectory the optics will collect the energy to the same spatial location where the photo-voltaic cell is positioned. Two novel designs are proposed while the first one is based on waveguides and the second on prisms with high concentration ratio of above 10X. The proposed configurations are validated numerically and experimentally.

[DOI: http://dx.doi.org/10.2971/jeos.2013.13033]

Keywords: Solar energy, concentrators

\section{INTRODUCTION}

Usage of solar energy for generation of heat and electricity recently became a very significant engineering as well as scientific trend [1]-[4]. As the cost of photo-voltaic (PV) cells is higher than the cost of the concentration optics it is financially worth developing concentration optics in order to minimize the area of the PV cells that will eventually concert the solar energy into electricity. There are two types of contractors: (a) Active concentrators that follow the sun and as they are always positioned in front of the sun they can reach very high concentration ratio of many hundreds or even thousands (this ratio is counted in units of Suns) [5]-[9]. The main disadvantage of those systems is that they require sun's tracking mechanics, due to high concentration factor they require cooling systems due to the heat dissipation and they do not work well in north countries where it is typically cloudy and thus in most of the days the sun light is not coming due to direct illumination but rather due to scattering from the atmosphere. (b) Passive concentrators that always look at the same direction and collect sun light from large angular range [10]-[13]. Their disadvantage is that usually they have very low concentration ratio of only 2 or 3 although recently a new design approaching concentration ratio of 10 was proposed [13]. The main advantage of the passive concentrators is their simplicity (do not require tracking mechanics) and their capability to function also in cloudy conditions.

In this paper we propose two novel passive configurations based on waveguides and on prism design. In Section 2 we present the theory behind the proposed idea and then construct the proposed designs and validate them numerically and experimentally in Section 3. The paper is concluded in Section 4.

\section{THE PROPOSED CONCEPT}

During the day-night time the sun crosses the sky along a 1-D trajectory. Between seasons the sun is also moving along a perpendicular 1-D axis resulting with overall 2-D rather than 1-D coverage of the space. The movement in the "summer-winter" axis of the movement trajectory is limited and does not cover more than about 46 degrees (twice Earth's axis tilt relative to its orbit). Taking into account this a priori information may result in increasing the obtainable concentration ratio as predicted from conservation of the Etendue (related to the law of brightness):

$$
n^{2} \int(\cos \theta) d \Omega \cdot d A=\text { const }
$$

In the last equation $d \Omega$ and $d A$ are the differential solid angle and the light collection differential area unit. $n$ is the refractive index and $\theta$ is the full angle between the lights direction of propagation and the normal to the inspected unit area (in paraxial approximation the term of $\cos \theta \operatorname{can}$ be assumed constant). This equation states that if we wish to concentrate light i.e. to decrease the area between the input and the output planes of our concentrator, we need to increase the distribution of the incoming light's solid angle $\Omega$ and this can be done only if to begin with the original angle $\Omega$ was sufficiently limited to allow its increase. All this is of course only if we do not want to lose energy (obviously one can concentrate by any desired factor if he does not care about losing energy).

If we use the last equation one can derive the concentration ratio as follows: As it is known from spherical coordinates, the vectorial differential length equals to:

$$
d \bar{l}=d r \hat{r}+r d \theta \hat{\theta}+r(\sin \theta) d \phi \hat{\phi}
$$


where $\hat{r}, \hat{\theta}, \hat{\phi}$ are vector units along the radial and the two angular directions respectively. $d r, d \theta$ and $d \phi$ are the differentials of the three spherical coordinates Thus, the differential solid angle can be expressed:

$$
d \Omega \equiv \frac{\text { DifferentialArea }}{r^{2}}=\frac{r d \theta r \sin \theta d \phi}{r^{2}}=\sin \theta d \theta d \phi
$$

Thus according to Eq. (1) the area compression ratio (the ratio between the overall input and output area of the optical module: $\Delta A_{\text {input }} / \Delta A_{\text {output }}$ ) is as described below by $\eta$ in Eq. (4), while in the input to our optical system we assumed $n=1$ (air) and angular range of $\Delta \theta$ and $\Delta \phi$ and after the output from our system (where the PV cell is located) we assumed refraction index of $n$ which is not 1 and maximal possible angular range of $\Delta \theta=\Delta \phi=\pi$ :

$$
\begin{aligned}
& \eta=\frac{\Delta A_{\text {input }}}{\Delta A_{\text {output }}}=\frac{4 n^{2} \int_{0}^{\pi / 2} \int_{0}^{\pi / 2} \cos \theta \sin \theta d \theta d \phi}{4 \int_{0}^{\Delta \theta / 2} \int_{0}^{\Delta \phi / 2} \cos \theta \sin \theta d \theta d \phi} \\
& =\frac{n^{2} \int_{0}^{\pi / 2} \int_{0}^{\pi / 2} \sin 2 \theta d \theta d \phi}{\int_{0}^{\Delta \theta / 2} \int_{0}^{\Delta \phi / 2} \sin 2 \theta d \theta d \phi}=\frac{\pi n^{2}}{\Delta \phi(1-\cos \Delta \theta)}
\end{aligned}
$$

We adapt Eq. (4) for the movement trajectory of e.g. $\Delta \phi=90^{\circ}$ "day-night" movement during which there is a non negligible amount of sun light and $\Delta \theta=40^{\circ}$ for the summer-winter movement. We also assume at the output of the concentrator refraction index of $n=1.7$ (reached in some polymers and glasses) and we obtain a maximal concentration ratio of around 25 Suns.

In this paper we will present two novel passive concentration designs:

- In the first we intend to obtain passive collection of light that is brought from the same optics to the same PV by plurality of waveguides while each waveguide is collecting energy in different seasonal period. Since the waveguides are very low in their price there is no problem in their replication. The optics itself and the PV cells are not replicated as all waveguides arrive to the same small area PV cell. By using such a solution the law of brightness is conserved and efficient passive collection of light is realized. The sun light is inserted into this array of planar and flexible plastic waveguides (allowing flexibility in the positioning of its output) via an array of tapered cylindrical cones. Due to the fact that the taper is a cylindrical lens, any movement of the light source along the axis that is perpendicular to the focusing axis of the lens is still well coupled into the planar waveguide. Combining several planar waveguides as such, and bringing them to the same PV allows collection of sun's energy by the same PV for various spatial locations of the sun.

Each waveguide is designed such that it is capable of collecting the energy from a light source with spatially varied location while the source is moving in one axis 180 degrees and e.g. up to 12 degrees in the other axis (the waveguides are planar and the coupling of light into them is done with a tapered cylindrical lens). 4 waveguides are coupled to the same tapered cylindrical lens and guide the collected light to the same PV cell. If, as described in our example, each one of the 4 waveguides collects light in different segment of 12 degrees (along the axis perpendicular to the axis of the focal length of the cylindrical lens) an overall coverage of 48 degrees is feasible for the movement of the sun along the summerwinter axis (which is, as mentioned above, should be enough as along the summer-winter axis the sun moves only along 46 degrees). Thus, when the position of the sun is varied due to seasonal changes each time a different waveguide (out of the mentioned 4) is carrying the collected energy from the same tapered lens to the same PV cell.

The solution of usage the plurality of flexible plastic waveguides is also good in the sense that the proposed system is very modular and by proper wiring of the waveguides any configuration of collecting optics and PV array can be matched (i.e. matching between the dimensions of the collecting optics and the PV cells). In addition the system is now tolerant also to the distance or the absolute location of the optics in respect to the PV cells array (the collecting optics may be placed on a roof of a building while the PV cells in the basement protected from the environmental conditions). The planar waveguides based solution may also allow realization of spectral splitting by adding proper processing of the surface of the waveguides [14].

- Prismatic wide angle sun concentrator based design in which we aim to perform an improvement of the basic waveguide based concept in sense of simplifying its fabrication complexity. This concentrator is based on an effect of total internal reflection inside of a prism. As at total internal reflection all the energy of the optical beam is repeatedly reflected without losses inside of the proposed special prism.

\section{EXPERIMENTAL AND NUMERICAL INVESTIGATION}

\subsection{Waveguide based concentrator}

We have constructed a single tapered lens coupled to 4 waveguides as well as an array of such tapered lenses coupled each to a set of 4 waveguides. The array allows increasing the area of the light collecting optics. In Figure 1(a) we present a constructed laboratory demonstrator of an array of devices while in Figure 1(b) one may see a single collector.

Note that one of the advantages for the proposed technology is the flexibility in adapting the collection architecture to the one of the PV cells. For instance, if the dimensions of the PV cells are different than those of the collection optics, the waveguides can be folded to match the PV architecture. This is well demonstrated in the image of Figure 1(b) where the folding appears in the upper right part of the figure. In Figure 1(c) we experimentally illuminate the device with green laser whose position is varied along 1-D axis and in all cases 


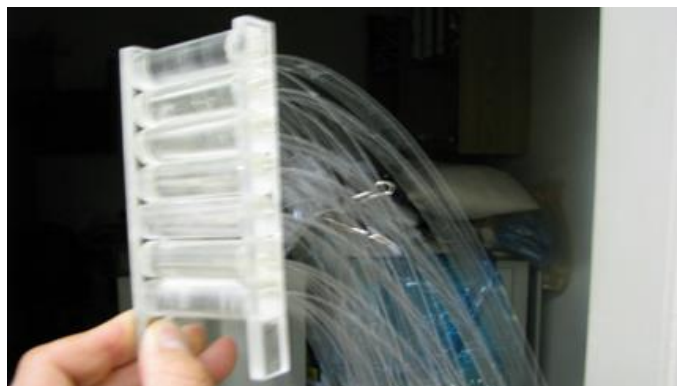

(a)
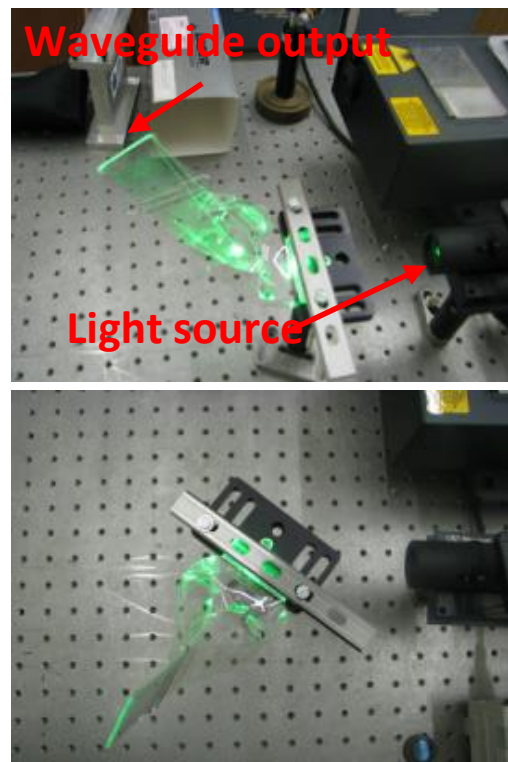

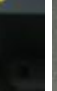

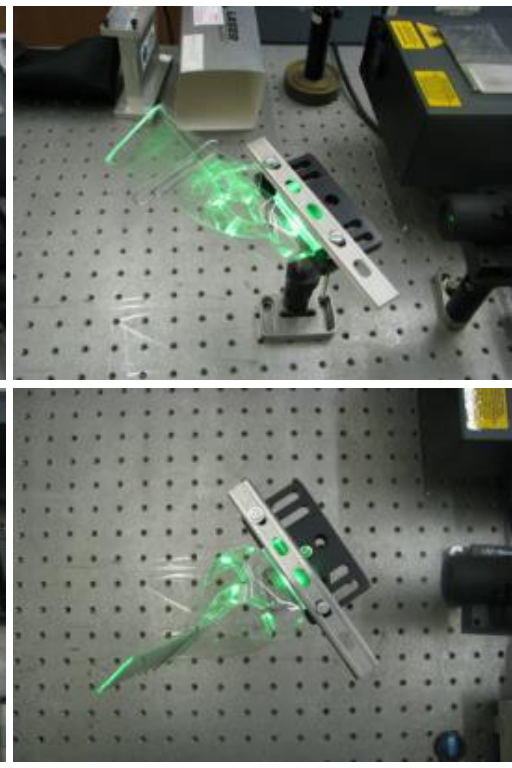

(c)

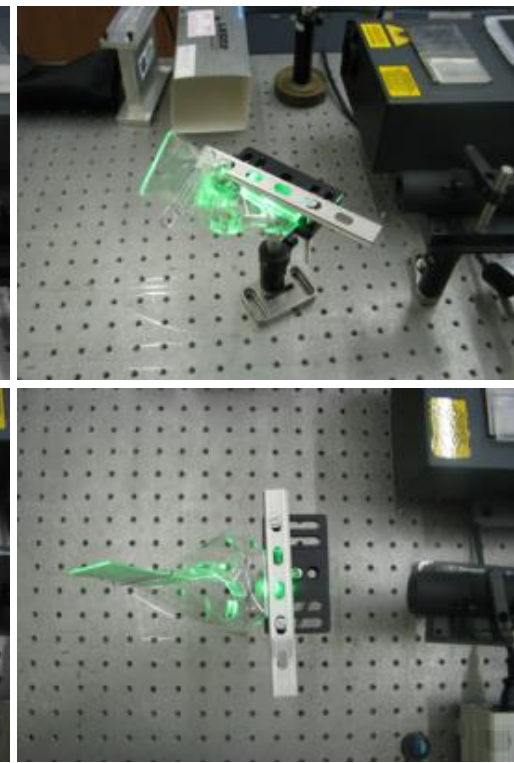

(b)
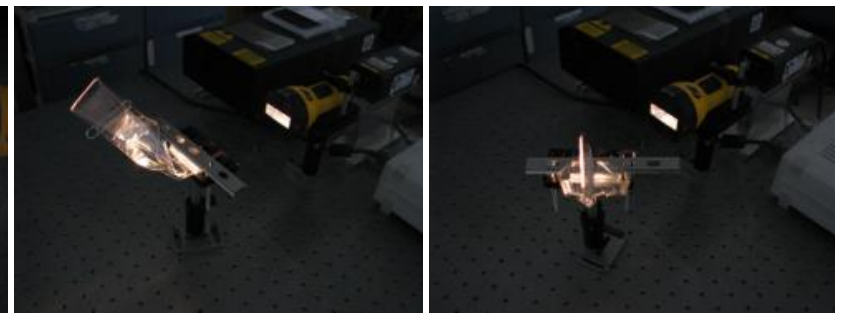

(d)

FIG. 1 (a). An array of special collectors. (b). An image of a single collector. (c). Experimental results, done with green laser, that demonstrate the invariance to the direction of the laser in the process of the energy collection. (d). The same experiment after being repeated with white light source.

the light is coupled into the waveguide and brought to a desired position in space (i.e. in Figure 1(c) the light comes from right to left and the flexible waveguide is bended to bring the coupled energy upwards). In Figure 1(d) we repeat the same demonstration with white light illumination.

The experimental results of Figure 1 fit to energy collection for 1-D sun's movement. In Figure 2 we demonstrate the experimental results for 2-D movement for the prototype of Figure 1(b). In Figure 2(a) we show the prototype for passive sun light collection for 2-D movement of the sun. Here we had every tapered cylindrical lens coupled to 3 planar waveguides each capable of supporting 2 degrees in the axis perpendicular to the axis of the focal length of the lens (i.e. perpendicular to the plane of the waveguide), as we wanted only to demonstrate the basic principle of operation.
In Figure 2(b) and 2(c) we demonstrate the lack of sensitivity to the direction (in the axis of the focal power of the cylindrical lens) of the illuminating source (for green light) while in Figure 2(b) we present -3 degrees of tilting and in Figure 2(c) it is +3 degrees of tilting (i.e. demonstrates the movement of the sun along the "summer-winter" axis). One may see the white arrow mark showing how the light is shifted from one waveguide to another waveguide due to the change in the tilting. Figure 2(d) is an overall image of the device. In Figure 2(e) we repeat the same experiment but with a white light source. As mentioned above, in this experiment we used 3 planar waveguides with capability to couple light at angular range of 2 degrees in the axis perpendicular to the plane of the waveguide.

Experimental results testing the uniformity of the energetic collection versus the "day-night" axis are presented in Figure 3(a). The experimental results validate the theoreti- 


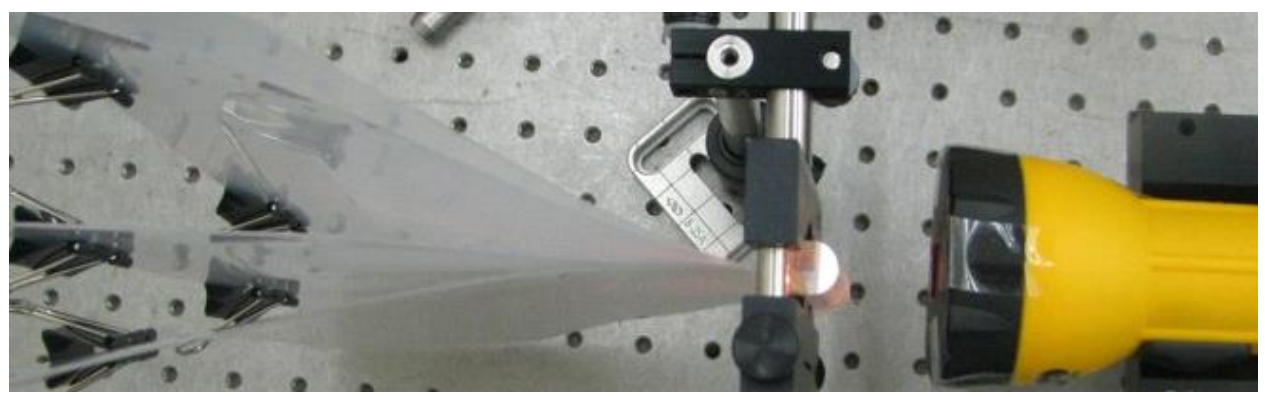

(a)

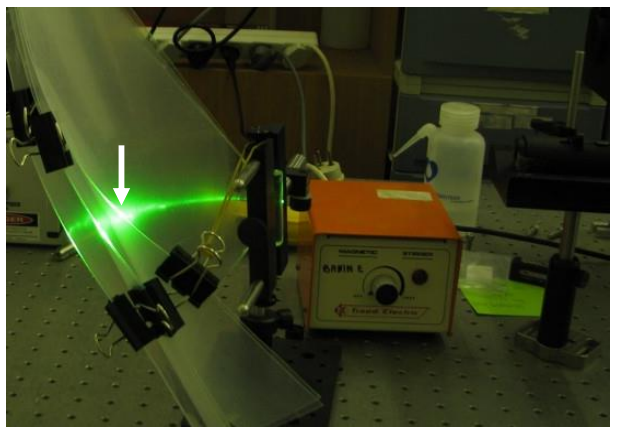

(b)

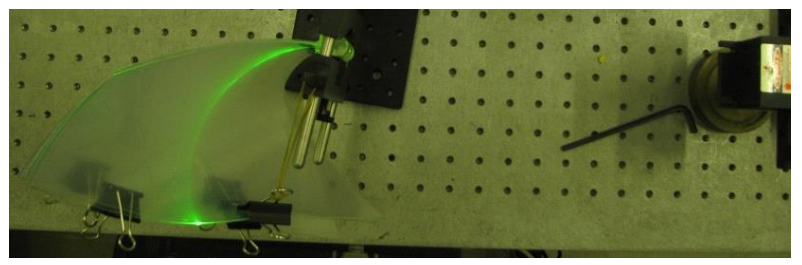

(d)

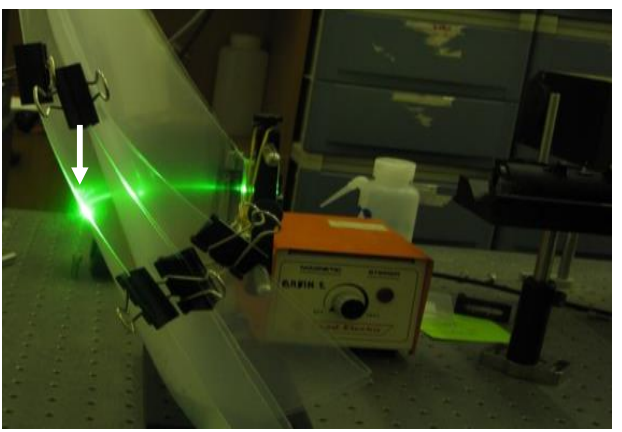

(c)

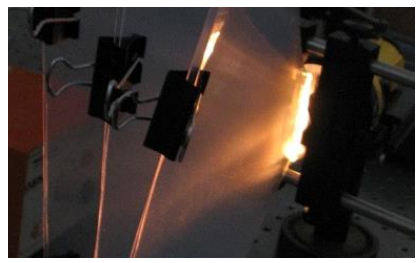

(e)

FIG. 2 (a). Prototype for passive sun light collection for 2-D movement of the sun. (b).-(d). Lack of sensitivity to the direction (along the axis of the focal power of the lens) of the illuminating source for green light. (b). -3 degrees of tilting. (c). +3 degrees of tilting. (d). Overall view of the device. (e). The same as (d) but for white light illumination.

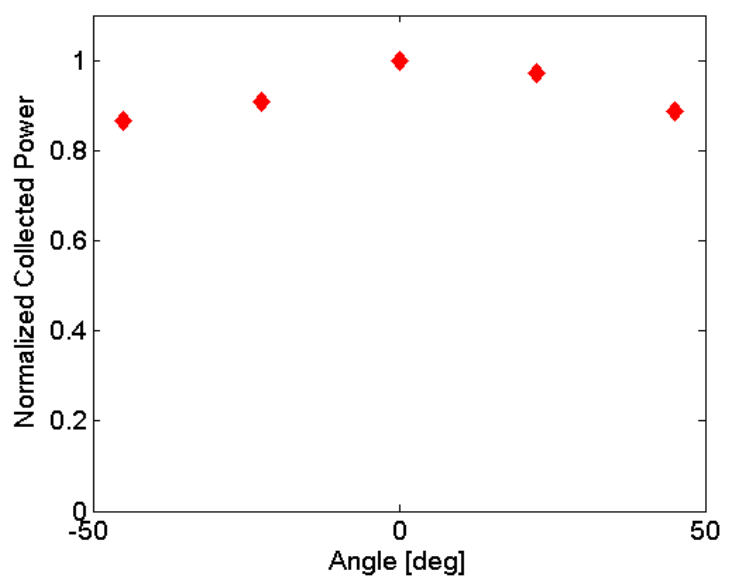

(a)

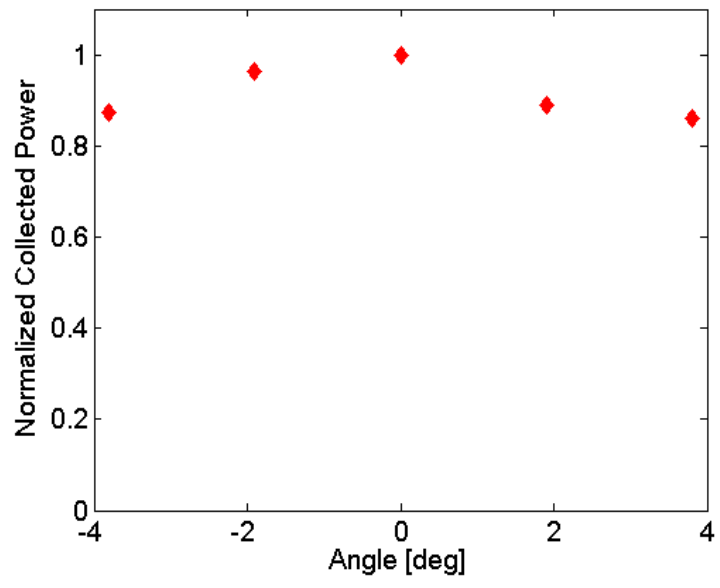

(b)

FIG. 3 Experimental results testing the uniformity of energetic collection versus (a). "day-night" axis. (b). "summer-winter" axis.

cally predicted uniformity of the energetic collection versus the "summer-winter" axis. The results are presented in Figure 3(b).

Concentration factor of around 20 Suns was measured as this was the $n^{2}$ times the ratio between the input face and the output faces to the constructed device.

\subsection{Prismatic concentrator}

An improvement of the basic concept previously explained is related to simplification in the fabrication complexity. The simplification is obtained by proposing a prismatic wide angle sun concentrator. This concentrator is based on an effect of full internal reflection inside of a prism. As at total internal reflection all the energy of the optical beam is repeatedly reflected without losses inside of the proposed special prism. 


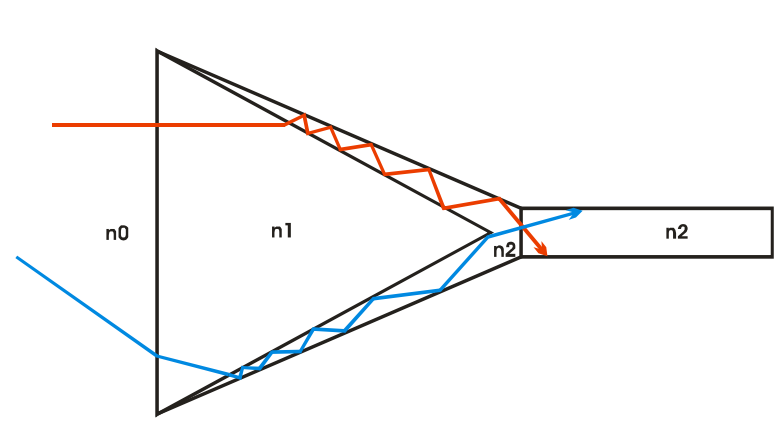

(a)

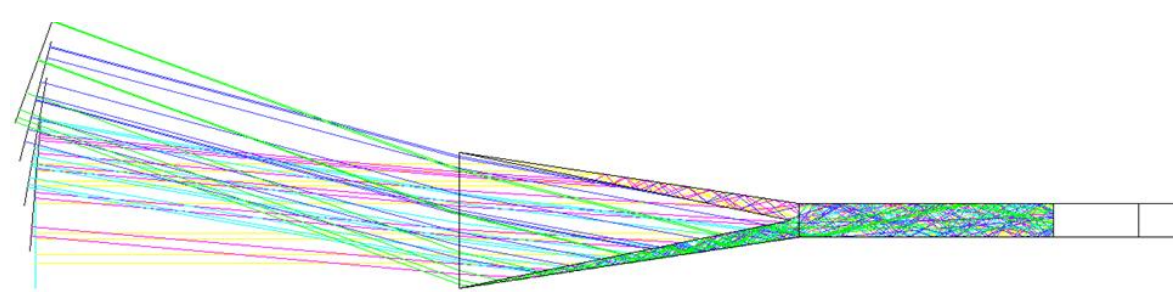

(c)

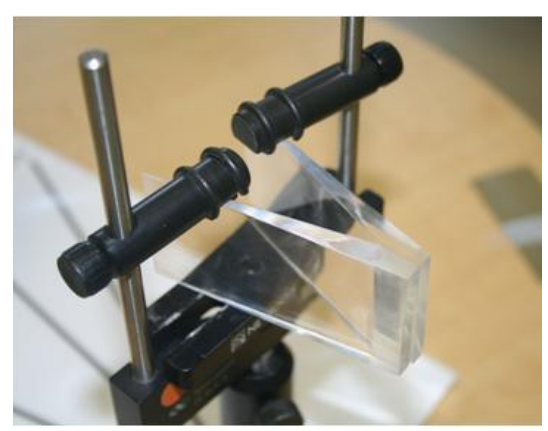

(b)

FIG. 4 (a). Schematic sketch of the prismatic concentrator. (b). Image of the constructed prototype. (c). Its numerical simulations.

The purpose of the prism is to increase the angular span of angles $\Delta \Omega$ in order to accordingly decrease $\Delta A$ (i.e. to concentrate light) as required according to the law of brightness specified in Eq. (1).

The schematic sketch of the proposed device is seen in Figure 4(a). The entrance beam is refracted from the border between materials having refraction indexes of $n_{0}$ and $n_{1}$ respectively. This refraction (Snell law) leads to reduction in the propagated angular range. Then the beams fall on a prism (border between materials with refraction indexes of $n_{1}$ and $n_{2}$ respectively). The fabricated corner and the region with refractive index $n_{2}$ of a prism are responsible to pick up the beams that have been reflected from the external border of a prism (border between materials with refraction indexes of $n_{2}$ and $n_{0}$ ) and to guide them via total internal reflection inside the prism having refraction index of $n_{2}$. Image of the constructed prototype is seen in Figure 4(b). Simulation of the improved light collecting configuration is presented in Figure 4(c). The simulation is performed for input angular range of 0 degrees and up to +20 degrees. But since the design is symmetric, the same result is expected also for angular range from - 20 degrees to 0 degrees. The obtained concentration factor in this 1-D simulation is 4 . The applied refractive indexes were $n_{0}=1$ (air), $n_{1}=1.437$ (Schott FK54 glass) and $n_{2}=1.952$ (Schott SF59 glass).

The proposed simulation is performed in 1-D (along the axis of "summer-winter"), in the second dimension (along the axis of "day-night") a full range of 180 degrees is allowed and thus there the concentration factor is coming only due to the higher refractive index (Snell law). Thus, in 2-D the overall concentration will be about a factor of $8(=4 \times 1.952)$. Note that this is not the most optimized design but rather a demonstration of an operation principle.

As mentioned above the proposed simulation and design of Figure 4 were for 1-D case (along the axis of "summer- winter"). In Figure 5 we present a 2-D design base on the same total reflection of the prismatic concept but optimized for 2D. In Figure 5(a) we show the numerical simulations of the improved prismatic 2-D sun concentrator (done with Zemax software for optical design) and in Figure 5(b) we show the constructed first laboratory prototype. It is constructed from Perspex and filled with water in its internal section (the blue region of Figure 5(b)). In the right part of Figure 5(b) we show how the prototype is being constructed. A PV cell was glued at the output face of the concentrator and its output voltage was measured. The use in water and in Perspex was done in order to generate the required difference in refraction indexes $\left(n_{1}\right.$ in respect to $n_{2}$ ). In Figure 5(c) we show some preliminary experimental characterization showing that indeed uniform energetic output is obtained over the predefined angular range of 40 degrees in the "summer-winter" axis and 140 degrees in the "day-night" axis. The experimental results are measured only for half of the angular range since there is symmetry to the other half. In Figure 5(c) we see both the 2-D plot of the measured voltage (left side) as well as its 3-D mesh (right side). A uniform readout versus the examined wide angular range was demonstrated. Concentration factor of 10 Suns was measured as this was the $n^{2}$ times the ratio between the input face and the output faces to the device.

In addition to the experimental demonstration of the obtained energetic uniformity over the above specified angular range, we have performed also measurements of the concentration and the energetic efficiency. Efficiency of close to $90 \%$ was measured. The measurement was performed with calibrated power detector.

\subsection{Numerical evaluation}

At first we wished to examine the dependence of the light concentration performance of the waveguiding design on wavelength shift that may occur due to material properties for the waveguide material as well as the effect of its fold- 

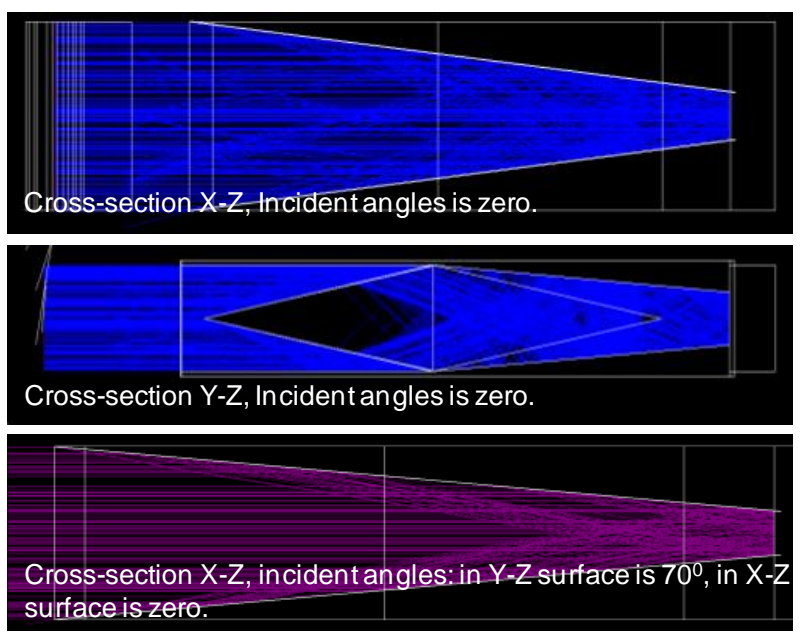

\section{surface is zero.}
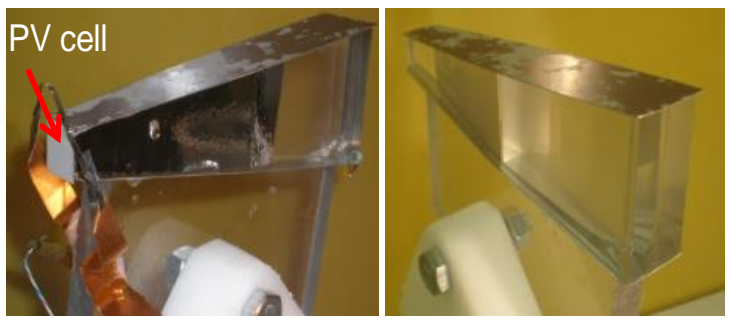

Improved prismatic sun concentrator. The second prototype capable of reaching 10 sun.

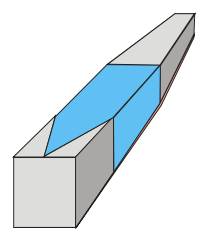

(b)
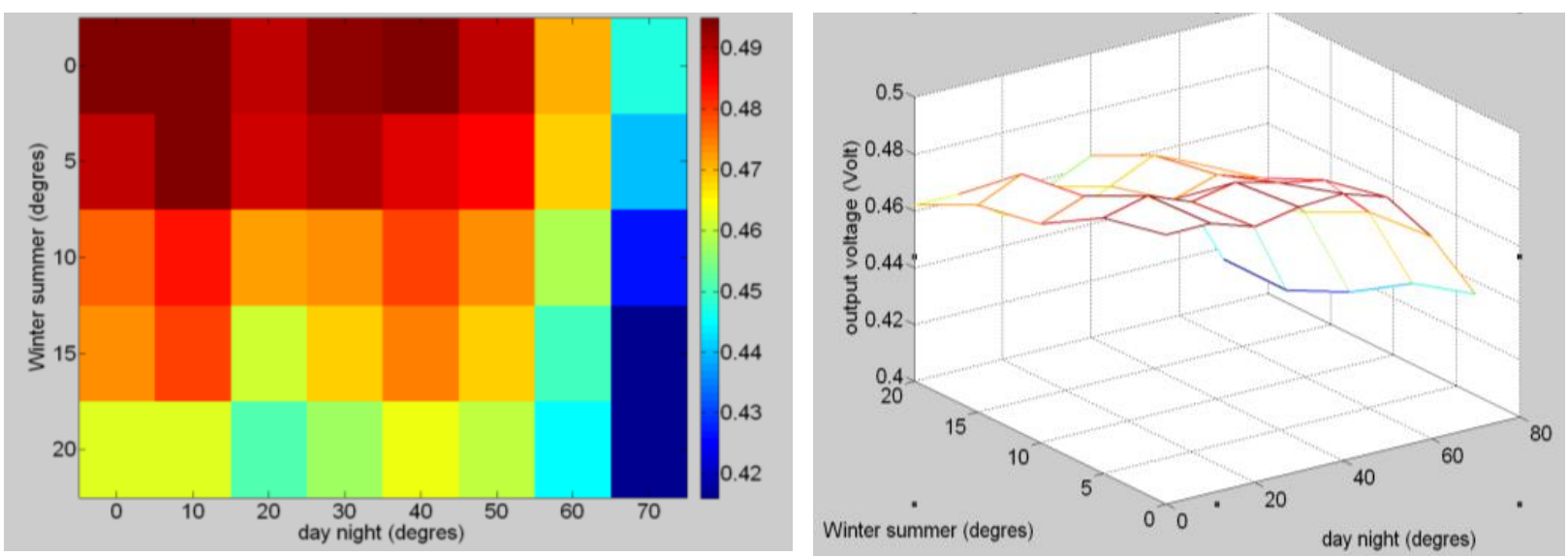

(c)

FIC. 5 (a). Numerical simulations for the improved 2-D prismatic sun concentrator. (b). The constructed laboratory prototype. (c). Preliminary experimental results.

ing. Folding of the waveguides is restricted in the sense of guiding strength and it might be wavelength dependent. In the numerical Zemax simulations presented in Figure 6 we examined both effects.

In Figure 6(a) we present the Zemax model of the waveguide folded at cone angle of 85 degrees with 3 wavelengths injected into its left side: $486 \mathrm{~nm}, 587 \mathrm{~nm}$ and $656 \mathrm{~nm}$. In the lower left side we see the 3D plot of the waveguide and the simulation itself shows its side view and the propagation of rays at the 3 colors through it. The number of rays lost due to the folding and due to the fact that 3 different wavelengths were used was less than $1 \%$. At folding with cone angle of 70 degrees no losses were observed at all for all 3 wavelengths.

In Figure 6(b) we present the cross section of the waveguide in the input and in its output face when a point source at 3 colors is injected in. One may see that for all 3 wavelengths at the output face the spot of light is extended according to the cross section shape of the waveguide (the same occurs in the prismatic design) but the output spot is not very much wavelength dependent.

At this point it is also important to note that in respect to the concentration performance on alignment and positioning of $\mathrm{PV}$ cell in both waveguide and prism configurations, the sensitivity in the lateral direction is very small as the spot obtained in the output (as well as area of the PV cell itself) is large. Nevertheless the PV cell should be glued to the output face of the concentrator for both designs, as the sensitivity of performance on the axial position of the cell is not negligible due to diffraction effects. 


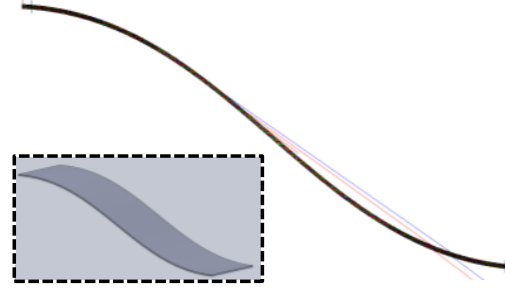

(a)

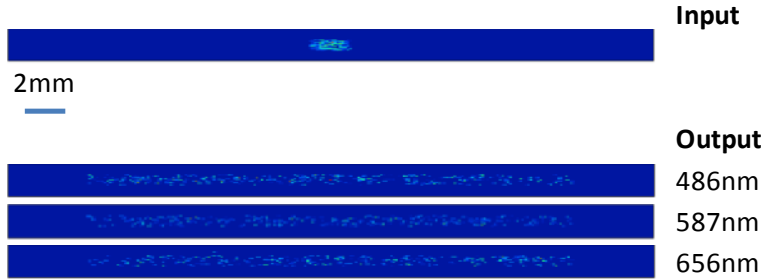

(b)

FIG. 6 Numerical Zemax simulations for the concentration performance dependence on wavelengths and on folding of the wave guiding design. (a). The Zemax model of the waveguide folded at cone angle of 85 degrees with 3 wavelengths injected into its left side. (b). The cross section of the waveguide in the input and in its output face when a point source at 3 colors is injected in. The output for each one of the 3 colors is presented separately.

\section{CONCLUSION}

In this paper we have presented two novel optical configurations for passive solar light concentration. Both designs are capable of producing high concentration ratio of above 10 Suns while properly exploiting the law of brightness and taking into account the a priori knowledge about the trajectory of the sun. Both configurations were validated numerically and experimentally.

\section{References}

[1] M. J. Currie, J. K. Mapel, T. D. Heidel, S. Goffri, and M. A. Baldo, "High-Efficiency Organic Solar Concentrators for Photovoltaics," Science 321, 226-228 (2008).

[2] J. S. Coventry, "Performance of a concentrating photovoltaic/thermalsolar collector," Sol. Energy 78, 211-222 (2005).

[3] C. Rotschild, M. Tomes, H. Mendoza, T. Carmon, and M. Baldo, "Luminescent Solar Concentrators: From Optical Heat Pumps to Solar Pumped Lasers," in Frontiers in Optics 2010/Laser Science XXVI, OSA Technical Digest (CD) (Optical Society of America, 2010), paper FMA1.

[4] W. G. J. H. M. van Sark, K. W. J. Barnham, L. H. Slooff, A. J. Chatten, A. Büchtemann, A. Meyer, S. J. McCormack, et al., "Luminescent Solar Concentrators - A review of recent results," Opt. Express 16, 21773-21792 (2008).

[5] A. Goldstein, and J. M. Gordon, "Double-tailored nonimaging reflector optics for maximum-performance solar concentration," J. Opt. Soc. Am. A 27, 1977-1984 (2010).
[6] J.-H. Huang, W.-C. Fei, W.-C. Hsu, and J.-C. Tsai, "Solar concentrator constructed with a circular prism array," Appl. Opt. 49, 4472-4478 (2010).

[7] V. C. Coffey, "Solar Concentrators: Using Optics to Boost Photovoltaics," Opt. Photonics News 22(1), 22-27 (2011).

[8] B. L. Unger, G. R. Schmidt, and D. T. Moore, "Dimpled Planar Lightguide Solar Concentrators," in International Optical Design Conference and Optical Fabrication and Testing, OSA Technical Digest (CD) (Optical Society of America, 2010), paper ITuE5P.

[9] J. H. Karp, E. J. Tremblay, and J. E. Ford, "Planar micro-optic solar concentrator," Opt. Express 18, 1122-1133 (2010).

[10] M. Hsieh, S. Lin, K. Y. Hsu, J. Burr, and S. Lin, "An Efficient Solar Concentrator using Volume hologram," in CLEO: 2011 - Laser Applications to Photonic Applications, OSA Technical Digest (CD) (Optical Society of America, 2011), paper PDPB8.

[11] N. Vaidya, R. Dauskardt, and 0. Solgaard, "AGILE: Axially Graded Index LEns as a non-tracking solar concentrator," in Renewable Energy and the Environment, OSA Technical Digest (CD) (Optical Society of America, 2011), paper JWD2.

[12] R. Winston, and W. Zhang, "Pushing concentration of stationary solar concentrators to the limit," Opt. Express 18, A64-A72 (2010).

[13] S. N. Kivalov, and R. Perez, "Non-Imagine Solar Stationary Concentrators with Using Combination of Prisms and Reflective Surfaces," in Optics for Solar Energy, OSA Technical Digest (CD) (Optical Society of America, 2010), paper SMB2.

[14] 0. Fixler, N. Bar-David, and Z. Zalevsky, "Spectral separation of sunlight for enhanced operability of photo-voltaic cells," J. Photonics Energy 1(1), 015501 (2011). 